\title{
Improved Ray Tracing Algorithm based on Virtual Source Tree
}

\author{
Lin Zhou ${ }^{1, a}$, Fang Luo ${ }^{2, b}$ and Longpin Yang ${ }^{3, c}$ \\ ${ }^{1,2}$ College of Communication and Information Engineering, Chongqing University of Posts and \\ Telecommunications, Chongqing 400065, China \\ ${ }^{3}$ Beijing Hostipal, Beijing 100005, China \\ aemail: 1058443685@qq.com, bemail: luofang199@gmail.com,,cemail:825908296@qq.com
}

Keywords: ray tracing; virtual source; depth-first; traversal

\begin{abstract}
Aiming at the low efficiency problem of the existing ray tracing algorithm, we design a low-complexity ray tracing algorithm based on the virtual source. In order to reduce the computational complexity, we design a concept-"virtual wall" to reduce the number of walls to be searched. Meanwhile, the depth-first-search algorithm is employed to complete the tree structure establishment of the virtual source, which can acquire all the effective paths between the source and the destination node without repeating traversal. The simulation results show that the improved algorithm can accurately find all the valid path from the transmitter to the receiver, and the. computational complexity of the improved algorithm is reduced.
\end{abstract}

\section{Introduction}

In recent years, with the development of mobile communications technology and communications services, the high demand for personal communications system capacity conflicts the limited resource of frequency. Microcell system using FDM (Frequency Division Multiplexing) to alleviate this contradiction has been widely used. In this process, on the one hand the effective coverage problems of radio waves have become increasingly prominent, on the other hand those experiential models obtained from statistical way will no longer apply. Ray tracing method based on geometrical optics theory (GTO) and uniform theory of diffraction (UTD) [1] is a prediction technique of radio wave propagation characteristics used in microcell environment [2,3].

Ray tracing method can be divided into two categories: forward and reverse ray tracing method. In forward method, infinite number of emitted rays are quantified into a limited number, In order to obtain the final valid paths, tracing each path to determine whether it can reach the receiving point. Forward method has a faster speed, but both radius of the reception ball and the quantizing step size of emission angle affect the accuracy of the algorithm. Reverse method adopts the image theory of geometric optics to seek multi-level image nodes so as to get the image tree, then tracing a path from the receiving point to the source point. However, high complexity exists in the reverse algorithm. Document [4] presented a ray tracing algorithm based on virtual source tree to get better prediction accuracy, however, this algorithm still exists the problem of low efficiency. For this reason, we design a low-complexity ray tracing algorithm based on the virtual source in order to reduce the computational complexity.

\section{Propagation modeling}

Propagation model is generally divided into two categories: 3D models and 2D models. The scenario this paper studies is urban micro-cellular environment, where transmitting and receiving antennas is less than the height of the surrounding buildings, in this case the diffraction from the top of the building to the receiving antenna can be ignored [5,6,7], thus, the ray-tracing prediction can be done in the two-dimensional map. The scenario we choose is depicted in Fig.1, which illustrates a plane view of the downtown core of Ottawa, Canada[8].

As to the ray tracing algorithm, all propagation paths are a combination of reflection and edge diffraction. As is explained in the ray tracing algorithm based on virtual source tree [9], reflective 
surface and the diffracted edge are recorded in the tree structure. In order to better explain the principles of the virtual source tree algorithm, first define three sources: transmitter, image and diffraction sources. Creating a virtual source tree structure and judging the validity of each source [10] is the key to this algorithm.

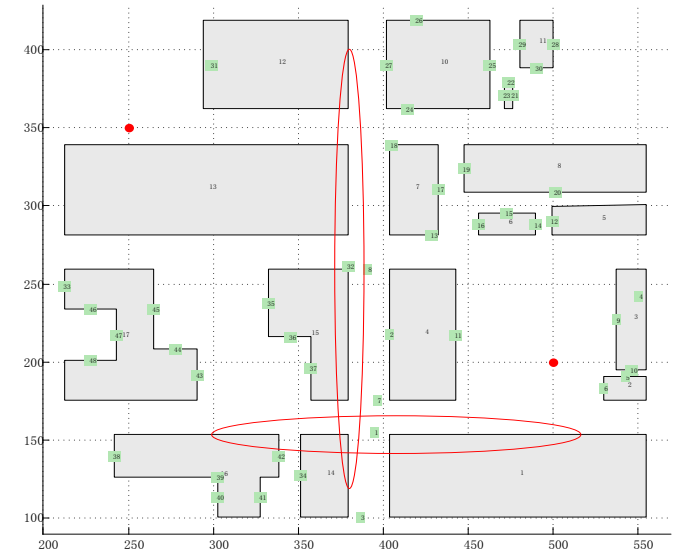

Fig. 1 Two - dimensional model of city building

\section{Accelerated algorithm based on virtual source tree ray tracing method}

Ray tracing algorithm based on the virtual source has high complexity, and the number of sources showed exponential growth with the reflective walls. In this paper, we propose the following two improvements: First, the aided design concept of "virtual wall" is adopted to reduce the number of walls to be searched, accomplish the validity decision of nodes, and compress the search space of the nodes.; meanwhile, the depth-first-search algorithm is employed to complete the tree structure establishment of the virtual source, which can acquire all the effective paths between the source and the destination node without repeating traversal.

Virtual wall: a "virtual merger" ideological of walls is adopted, the walls on the same line is regarded as the "same" (the ellipse shown in Fig 1), while the real wall is only the reachable surface of the "virtual wall". This is because the image nodes on these walls are coincident, based on which can eliminate the need for repeating the search. As shown in Table 1, the number of combined virtual walls was reduced by forty percent compared to the real wall, and the complexity of the algorithm is greatly reduced. In reality, the layout street of urban construction often has this smoothness, so the concept of a virtual wall has certain application value.

\begin{tabular}{|c|c|}
\hline Number of real walls & Number of virtual walls \\
\hline Eighty & Forty-eight \\
\hline
\end{tabular}

Table 1 Comparison of the number of walls before and after the virtual merger

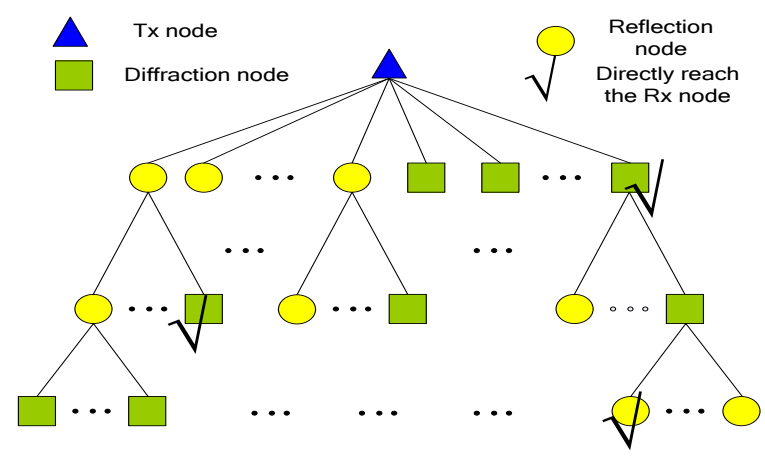

Fig. 2 Virtual source tree

When considering the diffraction, the effective diffraction node can be regarded as a virtual source, which can be added to the search tree. Here, the necessary and sufficient condition of validity is that it is shelterless between the diffraction point and the virtual source. On this basis, as 
is shown in Fig 2, we use a depth-first algorithm to create a virtual source multi-tree, each branch of the tree represents a path, if the current path can reach the receiving node, this path will be regarded as valid.

The steps of the accelerated algorithm is shown in Fig 3

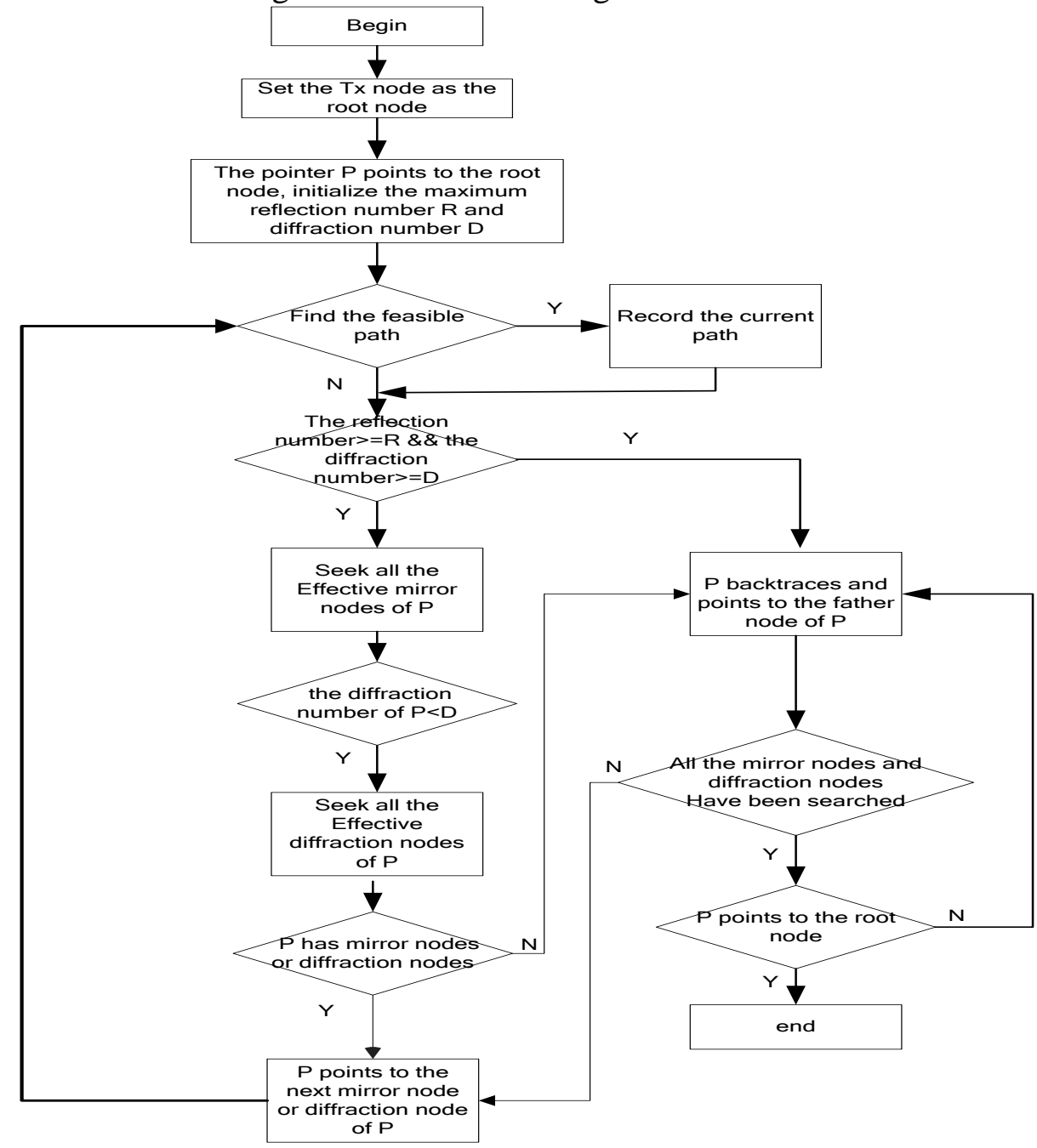

Fig. 3 Flow chart of the accelerated algorithm

\section{Simulation and analysis}

Select a model of buildings shown in Fig 1.Respectively set the coordinate of the emission point and the receiving point to be $(300,230)$ and $(500,200)$ to accelerate the simulation algorithm.

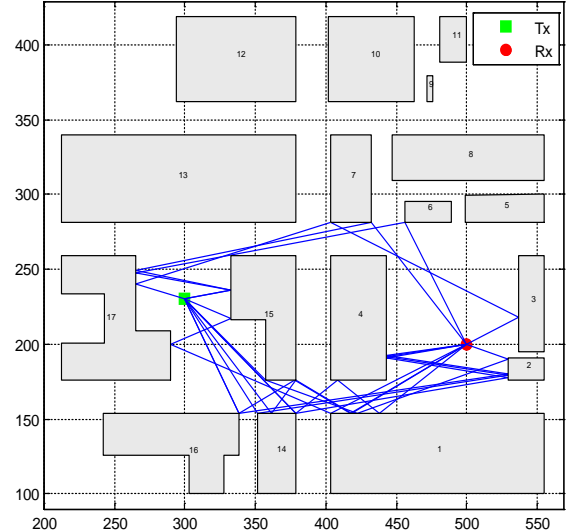

(a) Two reflections, one diffraction

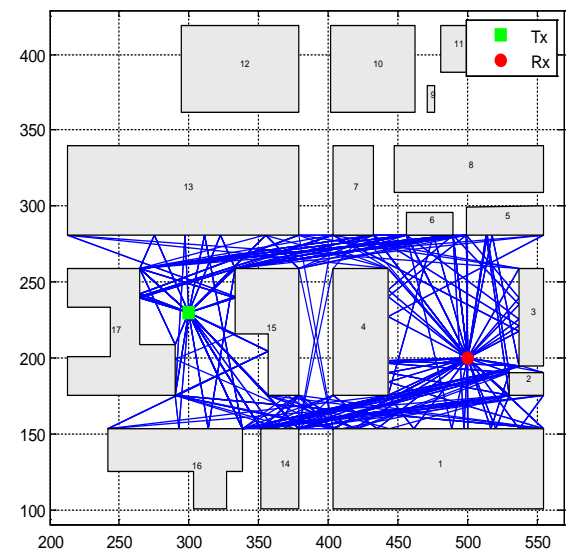

(b) One reflection, two diffractions

Fig 4 Comparison of two kinds of simulation 
As shown in Fig 4, the number of paths in Fig 4(a) is significantly less than in Fig 4(b), from which we can determine that when there is no direct path between the transmission node and the receiving node, the main propagation mechanism is diffraction.

\section{Summary}

To solve the high-computation-complexity problem of the ray tracing, we reduce the traversal number of the wall in the process of the validity judgement of the nodes with combining the virtual wall. Meanwhile the depth-first-search algorithm is employed to complete the tree structure establishment of the virtual source, which can improve the prediction efficiency of the algorithm. The simulation results show that the improved algorithm can accurately find all the valid path from the transmitter to the receiver, and this algorithm also can be used in complex urban environments.

\section{Acknowledgement}

The research work was supported by National Natural Science Foundation of China (Grant No.61171190).

\section{References}

[1] WANG Nan, LIANG Chang-hong, ZHANG Yu, et al. Study on the creeping ray-tracing algorithm of NURBS-UTD[J]. Journal of Xidian University, 2007, 34(4): 600-604.

[2] Song H B, Wang H G, Hong K, et al. A novel source localization scheme based on unitary esprit and city electronic maps in urban environments[J]. Progress In Electromagnetics Research, 2009, 94: 243-262.

[3] Gennarelli G, Riccio G. A uapo-based model for propagation prediction in microcellular environments[J]. Progress In Electromagnetics Research B, 2009, 17: 101-116.

[4] LIAO Bin, ZHAO Ni-li, ZHU Shou-zheng Ray tracing method based on a Virtual source tree [J]. Journal of East China Nomlal University(Natural Scicnce), 2008, 3: 103-108.

[5] Schettino D N, Moreira F J S, Rego C G. Efficient ray tracing for radio channel characterization of urban scenarios[J]. IEEE Transactions on Magnetics, 2007, 43(4): 1305-1308.

[6] Liu H, Li B, Qi D. Novel geometrical database model for line-based GIS urban maps in 2D/2.5 D ray-tracing algorithms[J]. Microwave and optical technology letters, 2004, 43(4): 307-310.

[7] Son H W, Myung N H. A deterministic ray tube method for microcellular wave propagation prediction model[J]. IEEE Transactions on Antennas and Propagation, 1999, 47(8): 1344-1350.

[8] Tan S Y, Tan H S. Propagation model for microcellular communications applied to path loss measurements in Ottawa city streets[J]. IEEE Transactions on Vehicular Technology, 1995, 44(2): 313-317.

[9] Feng Y S, Guo L X, Wang P, et al. Efficient ray-tracing model for propagation prediction for microcellular wireless communication systems[C]. IEEE 10th International Symposium on Antennas, Propagation \& EM Theory (ISAPE), 2012: 432-435.

[10] GU Xiao-long, ZHANG Wen-xun, YUN Zheng-ging et al. Improved ray-tracing technique with image approach based on visibility between objects[J]. Chinese Journal of Radio Science, 2001, 16(4): 464-467. 